\title{
Hybrid CMOS/Nanodevice Circuits for High Throughput Pattern Matching Applications
}

\author{
Fabien Alibart ${ }^{1}$, Timothy Sherwood ${ }^{2}$, and Dmitri B. Strukov ${ }^{1 *}$ \\ ${ }^{1}$ Electrical and Computer Engineering Department, University of California Santa Barbara \\ ${ }^{2}$ Computer Science Department, University of California Santa Barbara \\ *Email: strukov@ece.ucsb.edu
}

\begin{abstract}
We propose a class of novel hybrid CMOS/nanodevice circuits for pattern matching applications (e.g. real-time network intrusion detection, network packet routing, DNA sequencing), with the potential for dramatic improvements in throughput, density, and power performance relative to state-of-the-art designs. The performance advantage of our novel circuits is mainly due to three factors: the implementation of a ternary content addressable memory cell with stackable ultradense resistive switching ("memristive" or RRAM) devices; three dimensional hybrid CMOS/nanodevice circuitry with an area-distributed interface enabling high communication bandwidth between the memory and CMOS subsystems; and use of a modified CMOL FPGA fabric with low reconfiguration overhead.
\end{abstract}

\section{Introduction}

Pattern matching is a bottleneck operation in many important applications including network intrusion detection, network packet routing, DNA sequence matching, database searching, spell checking, spam filtering, and some image and signal processing tasks [1]. The primary performance metric for pattern matching tasks is typically throughput, which, for example, would be required to match the speed of communication network. Of course it makes little sense to discuss performance alone, and both energy per matching operation and latency are also important metrics in this space, (e.g. to enable fast and low power DNA sequencing [2] or minimize link delay in a filtering system).

At a high level, contemporary high performance approaches can be divided into two groups. The first approach makes use of the reconfigurable nature of field programmable gate arrays (FPGA), exploiting the fine grain configurability of the devices to implement a dense pattern matching structure [1-6]. For example, many
FPGA schemes make use of the configurable interconnect to stream data through a series of basic pattern matching operations performed by look up tables (LUT) inside logic blocks (Fig. 1). Going a step further, the reconfigurable nature of the hardware can be exploited to optimize the matching structures for the set of particular patterns being searched, e.g. through a technique analogous to common expression elimination $[5,6]$. The flexibility and bit-level configurability of FPGAs make them a natural platform for instance-specific highly parallel implementations in which both memory functions (i.e. storage of patterns) and logic operations are performed locally. On the other hand, reconfigurability comes at a high price, often with $40 \mathrm{x}$ increases in area and $3 \mathrm{x}$ increases in delay as compared to custom circuit implementations [7]. Pattern specific hardware reconfiguration can help to reduce these overheads, but this process is typically slow and limited by the peripheral $\mathrm{I} / \mathrm{O}$ bandwidth.

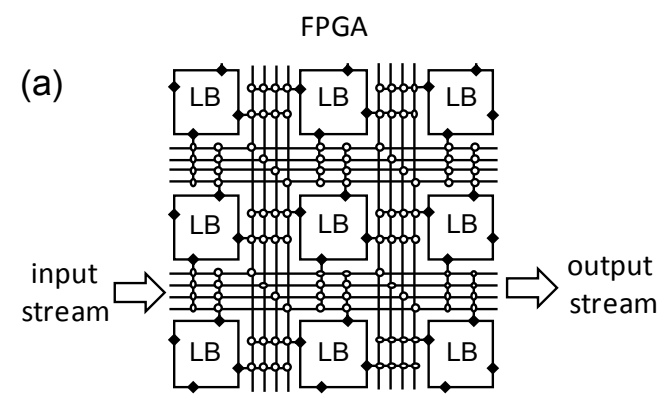

(b)

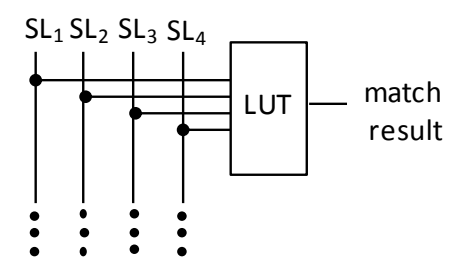

Figure 1. Pattern matching with island-type FPGA circuits: (a) general idea, and (b) matching operation performed inside one logic block (LB). 


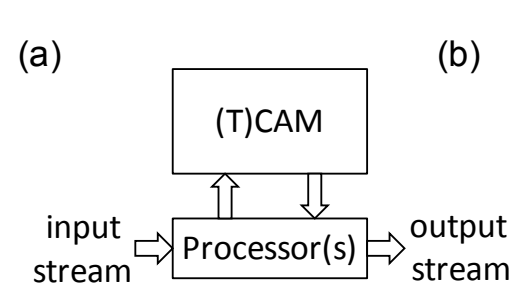

(c)

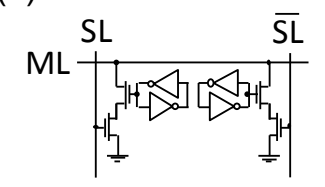

(d)

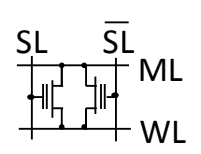

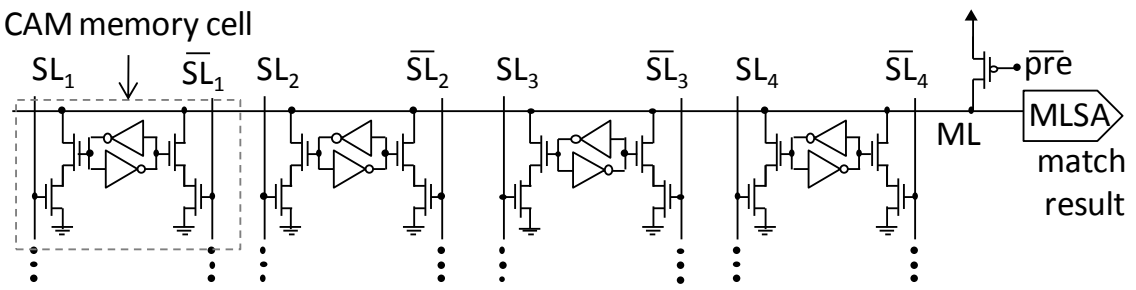

(e)

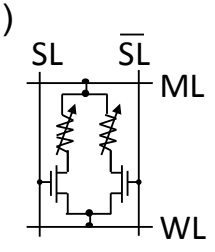

(f)

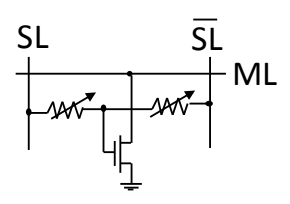

(g)

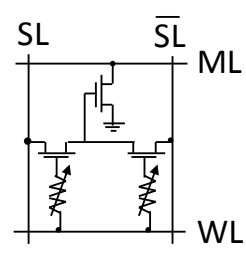

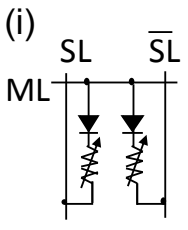

Figure 2. Pattern matching with content addressable memories: (a) general idea, (b) example of one row of SRAM-based CAM memory implemented with OR style [9], (c) SRAM-based TCAM memory cell [9], and (d-i) TCAM cells based on non-conventional technologies. In particular, panels $d, e, f$, and $g$ show TCAM cells based on flash memory [12], and hybrid CMOS/MRAM [14], CMOS/STT-RAM [11], and CMOS/memristors [13] technologies, while panel i shows implementation proposed in this work.

The second type of approach is based on ternary content addressable memories (T/CAMs) (Fig. 2) [8, 9]. These approaches rely on patterns being loaded into specially designed memories, which allow comparing a key from the input stream against stored pattern in massively parallel fashion (Fig. 2b). The dense structure of CAMs (roughly 2x larger than an SRAM cell) allows more patterns to be stored in the same unit of silicon, however the long memory lines used for matching must be charged and discharged on each and every search cycle, even when no matches are to be found.

Though both approaches are somewhat satisfactory for today's needs, looking forward, neither approach will scale particularly well to address future needs. For example, the inevitable development of faster $100 \mathrm{Gbps}-$ scale data networks, as well as increase of the number of patterns (e.g. the number of known viruses for network intrusion detection) will make real time protection an increasingly difficult prospect in the future [5] even using the most optimistic assumptions for scaling of complementary metal-oxide semiconductor (CMOS) technology [10]. A similar concern is for bioinformatics applications. The sizes of genomic banks have been exponentially increasing in the past decade making DNA sequencing and processing with conventional circuitry more and more challenging task [2].

It should be noted that several TCAM and CAM cells based on novel emerging technologies (Figs. 2c-g) have been suggested recently to improve the density of pattern matching circuitry [11-14]. Since all of these memory cell concepts involve the use of active circuitry (i.e. transistors), the scaling is likely to be limited to that of CMOS technology.

In this paper we are proposing novel hybrid CMOS/nanodevice circuits for pattern matching applications with significantly higher throughput and dramatically low energy per bit matched as compared to earlier suggested concepts.

\section{Novel hybrid circuits}

The advantages of the proposed circuits are mainly due the following three features: (i) ultra-dense diodelike stackable TCAM memory cell; (ii) high communication bandwidth three dimensional hybrid CMOS/nanodevice circuitry enabled by area-distributed interface (called CMOL [15, 16]); and (iii) modified CMOL FPGA circuit architecture [17-19] with low reconfiguration overhead. Let us now discuss more in detail these features.

\subsection{Diode-like stackable TCAM memory cell}

Novel TCAM cell (Fig. 2i) is based on a pair of nonlinear resistive switching ("memristive" [20-22]) devices. (Note that we also use terms "crosspoint device" and "nanodevice" in this paper to describe memristive devices). The inset in Figure 3c shows the simplest twoterminal memristive device structure consisting of two metal electrodes with metal oxide memristive layer sandwiched in between. 
(a)

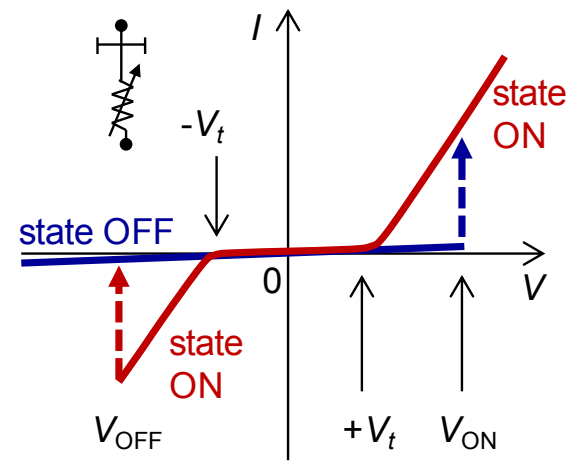

(b)

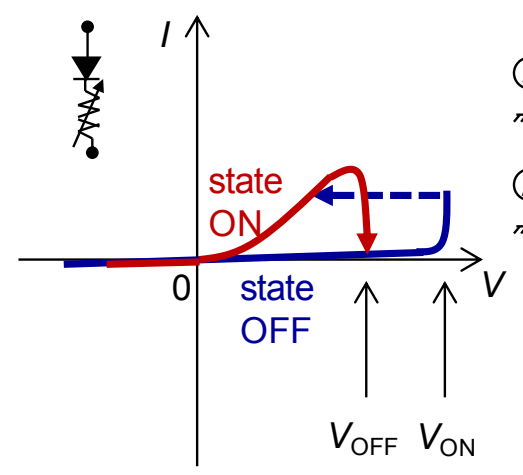

(c)

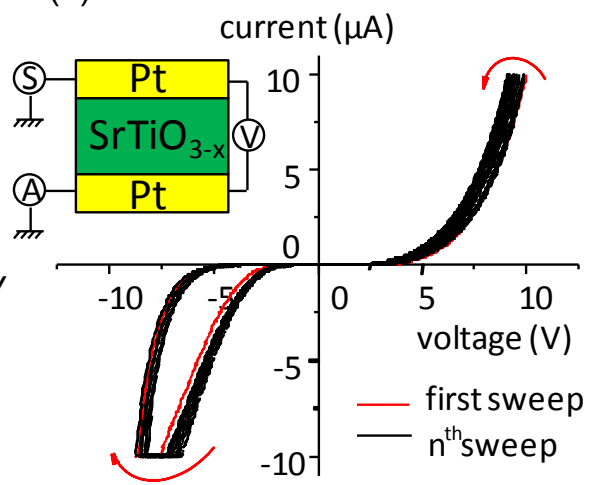

Figure 3. Resistive switching devices suitable for the proposed circuits: (a) bipolar device with symmetric I-V characteristics (shown schematically) with nonlinearity due to tunnel barrier, (b) unipolar device diode like I-V characteristics (schematically), and (c) our recent experimental I-V data for bipolar $\mathrm{SrTiO}_{3-\mathrm{x}}$ device. The device structure is shown in the inset of panel c with $\mathrm{S}, \mathrm{A}$, and $\mathrm{V}$ representing a voltage source, ammeter, and voltmeter.

Figure 3a shows typical $I-V$ for bipolar type of the devices which can be switched from low conductive state to high conductive one (called "on" switching) by applying positive voltage $V>V_{\mathrm{ON}}$. Once it is in high conductive state it can be switched back to low conductive state with voltage $V<V_{\text {OFF. }}$ The device state can be safely interrogated by applying voltages $V_{\mathrm{OFF}}<V$ $<V_{\mathrm{ON}}$. In contrast, unipolar devices are switched "on" or "off" with the voltage bias of the same polarity, though switching to the "on" state requires current compliance (Fig. 3b) to avoid oscillatory behavior.

Figure 4 shows an example of pattern matching operation with novel TCAM cells, in particular showing values which must be programmed to memristive device to detect "10X1" pattern in a stream of data. The matching operation is performed in two stages similar to conventional circuitry. First, match line (ML) is precharged to high voltage and then data values are supplied to signal lines (SL). ${ }^{1}$ During the second stage match line is not discharged only if values on signal lines are matching the programmed pattern, i.e. "1001" or " 1011 " in the considered example. On the other hand, if there is at least one mismatching value the match line will be discharged. Here it should be clear that nonlinear $I-V$ characteristics are essential for the operation in the second stage, i.e. it is needed to prevent charging of the match line via signal lines, and in fact the described pattern matching operation is performed by dynamic style diode logic. The required nonlinearity in the I-V characteristics can be achieved by either intrinsic properties of the

\footnotetext{
${ }^{1}$ In principle, a similar mode of operation is possible when match line is precharged to zero voltage and charged or unchanged during evaluation stage. In this case the current rectification direction in memristive devices should be reversed which can be facilitated with symmetric $I-V$ devices or reversing diode polarity.
}

device (Fig. 3), e.g. internal tunnel barrier gap [23] or Schottky barrier [24], or explicitly formed tunnel barrier [25] or diode [26].

The main advantage of the proposed cell is its high density and attractive scaling prospects. First of all, the cell structure is very compact because two-terminal memristive devices can be formed between two overlapping electrodes. If $F_{\text {nano }}$ is an electrode half pitch, the footprint of a TCAM cell (consisting of two memristive devices) could be as small as $2 \times 4 F_{\text {nano }}{ }^{2}$. Moreover, in contrast to field effect transistors, the operation of memristive devices is typically sensitive to only one critical dimension, i.e. thickness of the film (inset of Fig. 3c). As a result, the lateral dimensions, i.e. $F_{\text {nano, }}$, can be defined more aggressively, for example, using nanoimprint technology allowing to pattern sub-10$\mathrm{nm}$ features, i.e. far beyond resolution of conventional optical lithography.

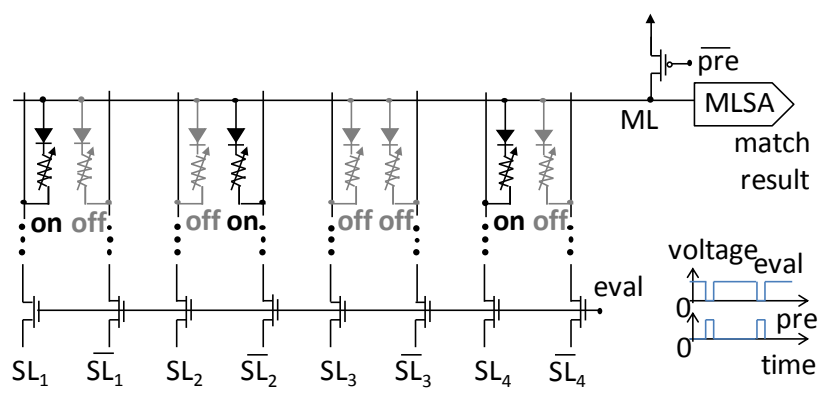

Figure 4. Example of memory configuration to detect pattern "10X1".

Finally, fabrication process for some devices does not involve high temperatures opening possibilities for monolithical back-end integration of multiple layers on 
CMOS substrate [16, 19]. Even if crossbar wires are defined with optical lithography so that $F_{\text {nano }}=F_{\mathrm{CMOS}}$, the effective footprint of the TCAM cell could be still very small, i.e. of the order of $8 F_{\mathrm{CMOS}} / K$, when $K$ memristive device layers are vertically integrated.

It is worth mentioning that, in general, any type of resistive switching effects and material systems could be employed to implement proposed TCAM cell, e.g. devices based on magnetic and ferroelectric tunnel junctions, phase change transitions, charge trapping in organic materials, solid state electrolytes, or transition metal oxides. However, the latter two memory concepts are the most attractive because of the combination of high $\mathrm{ON} / \mathrm{OFF}$ ratio, scaling prospects, and compatibility with CMOS [20, 21].

\subsection{D hybrid circuitry with area-distributed interface}

In principle, the proposed memory cell can be used to implement more efficient TCAM memory and pattern matching circuitry using approach shown on Figure 2a. While this approach should result in better performance over previously suggested pattern matching circuitry $[8$, $9,27]$ there are several issues which might prevent one from using such dense memory in the most efficient manner. These issues will be described next, along with our novel solution which is a hybrid approach combining advantages of TCAM and programmable circuits. In particular, one major feature of our approach, 3D hybrid circuitry with area-distribute interface, was presented earlier in the context of digital memories $[15,16,20]$, and we will briefly review it in this section. The other feature, dynamic CMOL FPGA, which is a modification of original CMOL FPGA circuitry $[17,18]$, will be explained more in details in the next section.

One of the main challenges is to sustain the density of memristive devices at the circuit scale. In particular the problems are, first, that memristive devices are passive, i.e. they cannot be used to amplify a signal. Secondly, the circuitry must ensure that each memristive device can be interfaced and programmed uniquely without negating the density advantages of nanodevices. The solution to these problems which comes with rather minor overhead is CMOL circuits $[15,16,20]$.

In CMOL memristive devices are integrated in the crossbar structures which are defined on top of the conventional CMOS substrate (Fig. 5). CMOS circuitry provides signal restoration and gain for logic circuitry and used to build decoders and sensing circuitry to program memristive devices. A unique feature of CMOL circuits is area distributed interface which enables high vertical bandwidth, and comes with potentially very low cost and low overhead. (Another property of such interfaces is that it relaxes overlay alignment requirement between nano and CMOS layers $[15,20]$ though this may not be important if both CMOS and crossbar layers are fabricated with conventional optical lithography). The area interface is enabled by (i) the crossbar array which is rotated by an angle $\alpha=\arcsin (1 / \beta)$ with respect to the mesh of CMOS-controlled vias; and (ii) a double decoding scheme that provides a unique access to each crosspoint memristive device. More specifically, as Fig. 5 shows, two types of vias, one connecting to the lower (shown with blue dots) and the other to the upper (red dots) wire level in the crossbar, are arranged into a square array of "basic cells" with sides $2 \beta F_{\text {Смоs. Here } \beta>1 \text { is a }}$ dimensionless number that depends on the cell size (i.e. complexity) in the CMOS subsystem. Note that with rotated crossbar array vias naturally subdivide the wires into fragments of length $2\left(\beta F_{\mathrm{CMOS}}\right)^{2} / F_{\text {nano. The factor } \beta \text { is }}$ not arbitrary, but is chosen from the spectrum of possible values $\beta=\left(r^{2}+1\right)^{1 / 2} \times F_{\text {nano }} / F_{\text {CMOS }}$, where $r$ is an integer so that the precise number of devices on the wire fragment is $r^{2}-1 \approx \beta^{2}\left(F_{\mathrm{CMOS}} / F_{\text {nano }}\right)^{2}$.

The decoding scheme in CMOL is based on two separate address arrays (one for each level of wire in the crossbar so that there are a total of $4 N$ edge channels to provide access to two different via controllers (one 'blue' and one 'red') in each of $N^{2}$ addressing cells in the CMOS plane (Fig. 5). In contrast to standard memory arrays, in CMOL each control and data line pair electrically connects the peripheral input/outputs to a via instead of a single memory element. In turn, each via is connected to a wire fragment in the crossbar. The two perpendicular sets of wire fragments provide unique access to any crosspoint device even for large values of $\beta$. For example, selecting pins $\delta \mathrm{v}$ and $\mathrm{b} 4$ (which are highlighted with blue and red circles, correspondingly) provides access to the leftmost of the two shown devices on Fig. 5a, while pins $\delta v$ and $\mathrm{c} 4$ for the rightmost device.

The total number of crosspoint devices that can be accessed by the $N \times N$ array of CMOS addressing cells is $\sim N^{2} \beta^{2}\left(F_{\mathrm{CMOS}} / F_{\text {nano }}\right)^{2}$, which may be much larger than $N^{2}$, if $F_{\text {nano }}<F_{\text {CMOs. }}$. An alternate way of looking at this fact is that one can use complex CMOS circuitry built with a significantly larger feature size to address regular crossbars built on a finer lithographic scale. Furthermore, the total number of memristive devices which can be accessed with double decoding scheme is $N^{4}$ >> $N^{2} \beta^{2}\left(F_{\text {CMOS }} / F_{\text {nano }}\right)^{2}$. More specifically, the first level of decoding selects two of $2 N^{2}$ vias with $4 N$ edge channels using a four-label address. The second level of decoding should, in principle, enable the selection of $N^{4}$ crosspoint devices using the $2 N^{2}$ internal lines (vias) of the area interface. This fact has been used to extend original idea 
(a)

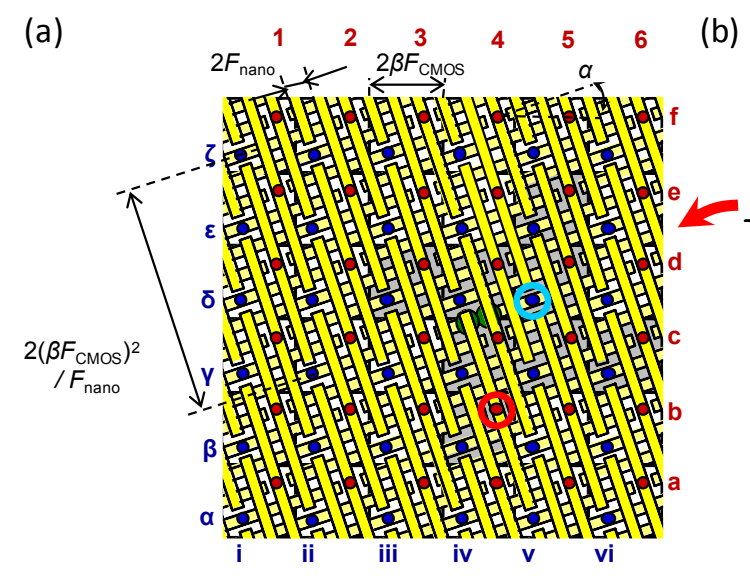

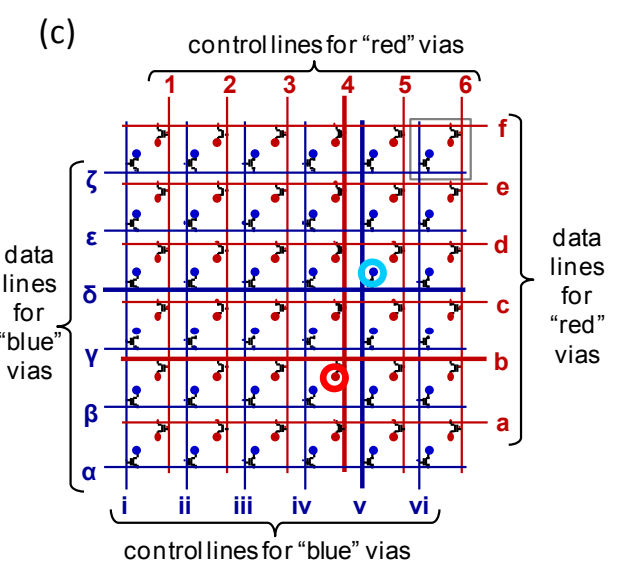

Figure 5. CMOL circuits [15]. (a) Cut-away illustration showing two types of vias connecting the CMOS control circuitry to the lower (blue) and upper (red) wire levels of the crossbar. (b) Top view of the crossbar structure showing $\alpha$ for $r=3$. (c) Corresponding equivalent circuit diagram of the configuration logic in CMOS layer for the $N=5$ primitive cell array. For clarity, panel b shows only two memristive crosspoint devices (green dots) which are used for the explanation in text.

to implement 3D CMOL circuits via stacking multiple crossbar arrays on top of each other using just one set of vias to connect all of the arrays to the cells [16]. The problem of stacking multiple layers becomes a geometric challenge to ensure that only one crosspoint device in all of the arrays can be addressed by any allowed set of four address labels (or a pair of vias). For example, one approach (out of many different possibilities) is to place the next crossbar in a sequence is to shift it with respect to the fixed locations of one kind of via by a distance such that the contacted wire fragments in the new layer are connected to a new connectivity domain that is different from any preceding layer.

It should be noted that the simplest way to build $3 \mathrm{D}$ CMOL circuits would be to use optical lithography to implement crossbar nanowires, i.e. with $F_{\text {nano }}=F_{\text {CMOS }}$, because it provides high accuracy of overlay alignment for all layers in the circuits. In this case, instead of implementing tilted crossbar, one can rotate the position of vias so that all metal lines satisfy Manhattan geometry [19].

\subsection{Dynamic CMOL FPGA circuit architecture}

One of the attractive features of CMOL is that CMOS circuitry can be customized to the particular needs of application, as long as it retains the basic circuitry serving regular array of vias shown on Fig. 5. In original CMOL FPGA circuits, the basic unit cell, which hosts two vias and two pass transistors, is augmented with CMOS inverter (with its input connected to via of one kind and output to another kind) to provide signal restoration and inversion [17]. In such fabric, one cell plays the role of configurable diode logic NOR gate. Its connectivity to other cells (and gate's fan-in) is specified by selectively programming to the on state memristive devices which are attached to the nanowire fragments connected to the inputs and the outputs of CMOS inverter. Because all configuration overhead in $\mathrm{CMOL}$ FPGAs, including all configuration memory and some routing circuitry, is lifted above the CMOS plane such circuits are estimated to yield up to two orders of magnitude density advantage when implementing typical benchmark circuits even for $K=1[17,18]$. These results, coupled with the recent experimental demonstration of CMOL FPGA-like circuits [28], support the feasibility of this approach. (Note that in more recent works, the logic fabric was built with more complex CMOS gates and in some versions memristive devices are only utilized for routing purposes $[18,28]$.)

Though the density advantage is significant, the memristive device utilization in reported work on original CMOL FPGAs is well below $1 \%$, due to the limited benefits of utilizing high fan-in gates $[17,18]$. The main contribution of this work is to show that a certain modification of the original CMOL FPGAs can dramatically increase memristive device utilization and turn them into very high bandwidth general purpose pattern matching circuits.

One modification to the original concept is that our approach is based on uniform fabric of CMOS D-flip-flop cells in which flip-flop is interconnected with the crossbar with the help of four vias. In particular two output vias (shown with red circle) are connected to normal and complimentary outputs of flip-flop and two inputs ones (shown with blue circle) are connected to D input (Fig. 6d). Clock signals are assumed to be routed using CMOS subsystem and are not shown in Fig. 6. During 
programming, CMOS flip-flop is disabled in all cells, e.g., by having "eval" CMOS line set to low. As a result any crosspoint memristive device (shown with green circle on Fig. 6c) in a crossbar structure can be setup to on or off state with the help of CMOS pass transistors and CMOS data and select lines, i.e. utilizing double decoding scheme of CMOL which was described above.

The second modification is dynamic style of logic similar to the pattern matching operation performed by TCAM circuitry considered above (Fig. 2). Such modification is necessary since static diode logic proposed in original CMOL FPGAs is not scalable for larger fan-ins. In particular, after the programming stage, logic operations are implemented with dynamic diode logic formed by ON-state memristive devices and CMOS pass transistors, while signal restoration, inversion, and latching is performed by CMOS D-flip-flop (Fig. 6). More specifically, the logical summation (i.e. Boolean "OR" operation) is performed in dynamic fashion in two stages during the clock period. During the first shorter stage the outputs of flip-flop in all cells are disconnected from corresponding crossbar wire using CMOS "eval" line. At the same time crossbar wire connected to the inputs and the outputs of flip-flop are precharged to high voltage using "select/pre" and "data/pre" CMOS lines. During the second stage, the "eval" signal is set to "high" voltage. This results in logical summation of the values from the cells connected to the particular crossbar wire with memristive devices turned to the ON state. At the rising clock edge, the value latched at the flip flop so that it can be used in unchanged or complimented form in the next clock cycle. Note that, though the operation performed on the crossbar wire is equivalent to OR Boolean operation, Boolean AND could also be performed in one cycle utilizing De Morgan's law due to the presence of complimentary outputs Q.

\section{Discussion and summary}

Let us now estimate performance of dynamic CMOL FPGAs. From the discussion so far it is clear that one flip flop cell can perform pattern matching in TCAM fashion in just one clock cycle (Fig. 6). The maximum size of a pattern is determined by the number of memristive devices attached to two input nanowires of flip-flop cell, i.e. quasi vertical ones on Fig. $6 \mathrm{c}$, which is equal to $2\left(r^{2}-\right.$ 1) for one crossbar layer (see discussion of that in the previous section) and $2 K\left(r^{2}-1\right)$ for CMOL circuits with $K$ layers.

The universal flip-flop cells could be also configured to perform Boolean logic operations, e.g., to perform logical operation with the results of pattern matching operation and/or to stream data, e.g. by forming very deep pipelines. For example, given array of flip-flop cells one can configure every second row of cells to stream data, from left to right on Fig. 6, and have remaining cells to perform pattern matching. With such mapping the maximum pattern matching capacity per one flip-flop cell is $N_{\text {bits }}=1 / 2 \times 1 / 2 \times 2 K\left(r^{2}-1\right)$. Here the first factor of $1 / 2$ reflects the fact that only half of flip-flop cells is involved in pattern matching operation (the other half is used for streaming), and the second one that only half of the memristive devices can be used for pattern matching in the simplest case (i.e. only memristive devices programmed to connect to flip-flop cells which streams data). With such mapping the effective density of patterns stored and processed is just $1 / 4$ of the maximum passive crossbar memory capacity.

We can also estimate the minimum clock cycle by assuming that all power is dynamic, because the static leakage could be neglected for the memristive devices with high on-off ratio. The maximum clock frequency $f$ can be found from the equation $C_{\text {wire }} V^{2} f_{\text {max }} / 2=p_{\text {max }} A_{\text {cell }}$, where $V$ is a voltage swing, $C_{\text {wire }} \approx 4 \times 2 F_{\text {nano }} \times K r^{2} \times C_{\text {seg }}$ is a total capacitance charged per cycle for one cell (i.e. of 4 nanowire segments with $C_{\text {seg }}$ capacitance per unit length), $p_{\max }=200 \mathrm{~W} / \mathrm{cm}^{2}$ is a typical manageable power consumption density [10], and $A_{\text {cell }}=2 \times\left(2 \beta F_{\mathrm{CMOS}}\right)^{2}$ is a flip-flop cell area. For example, assuming rather conservative values $V=1 \mathrm{~V}, \beta=10, K=1$ and using data from Ref. [17] for crossbar wire capacitance, $f_{\max } \approx$ $p_{\text {max }} F_{\text {nano }} /\left(K C_{\text {seg }} V^{2}\right) \approx 100 \mathrm{MHz}$ for $F_{\text {nano }}=3 \mathrm{~nm}$ and $F_{\mathrm{CMOS}}=45 \mathrm{~nm}$, and $f_{\text {max }} \approx 1 \mathrm{GHz}$ for $F_{\text {nano }}=45 \mathrm{~nm}$ and $F_{\mathrm{CMOS}}=130 \mathrm{~nm}$. The pattern matching throughputs per unit area, defined as $N_{\text {bits }} f_{\max } / A_{\text {cell }}$, corresponding to these two cases are $10^{19}$ and $10^{18} \mathrm{bits} / \mathrm{s} / \mathrm{cm}^{2}$, respectively, which is much higher than any reported state-of-the art concepts.

Also note that the proposed circuits could potentially offer much higher pattern capacity without any performance penalty. Because the number of storage elements in existing hardware-based pattern matchers is limited by the 2D chip area, they must be dynamically reconfigured to accommodate additional patterns that are beyond their storage capabilities. Dynamic reconfiguration is a relatively slow process, and if pattern matcher could not fit all the patterns the throughput for a fixed area will be considerably smaller than the ideal value. On the other hand, the bit capacity of the dynamic CMOL FPGA can be seamlessly increased by integrating more crossbar layers using the multilayer CMOL idea [24]. Thus, our architecture can support more patterns without any degradation in throughput.

In conclusion, we believe that the proposed circuits are very promising for pattern matching applications. It is worth mentioning that the current main challenge towards building such circuits is yield of today's memristive devices, even considering high intrinsic defect tolerance of CMOL FPGAs [17]. However, large scale industrial efforts on passive crossbar memories give hope that this issue might soon be resolved. 


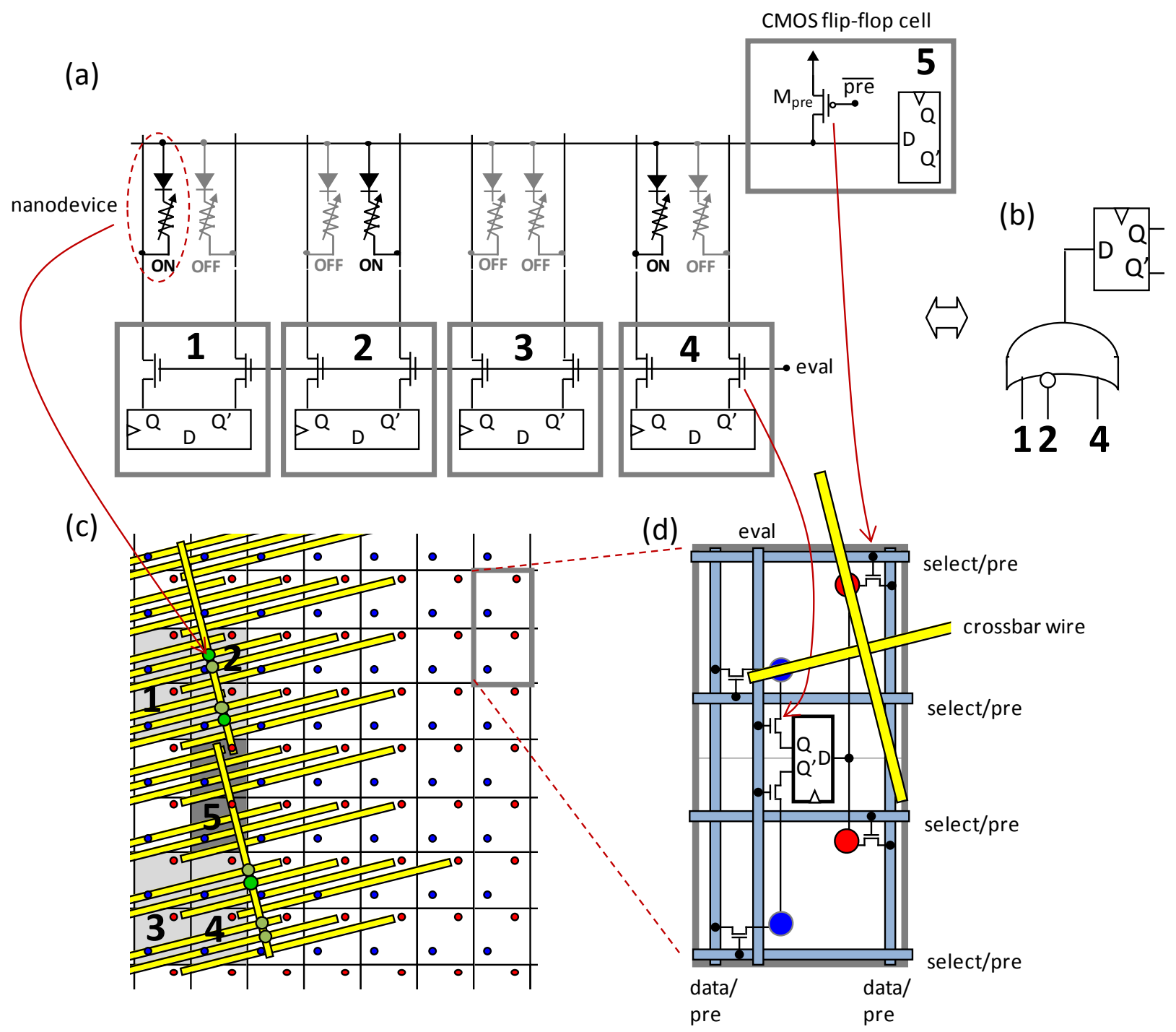

Figure 6. Dynamic CMOL FPGA logic: (a) Example of mapping of circuit from Figure 4 and (b) its equivalent functionality to (c) dynamic CMOL FPGA fabric which consists of (d) multipurpose D-flipflop cells. Note that for clarity panel $c$ only shows nanowires which overlap with input nanowires of cell denoted with number " 5 " and only those nanodevices which participate in the considered example. Bright and light green dots denote nanodevices in the on and off states, correspondingly.

\section{Acknowledgments}

The authors would like to thank K. Likharev, A. Mishchenko, and R. Brayton for useful discussions. The work is supported by NSF grant CCF-1017579.

\section{References}

[1] S. Hauck and A. DeHon, Reconfigurable computing: The theory and practice of FPGA-based computation. Boston, MA: Morgan Kaufmann, 2008.

[2] B. Schmidt, Ed., Bioinformatics: High Performance Parallel Computer Architectures. CRC Press, 2010.
[3] Z. K. Baker and V. K. Prasanna, "Time and area efficient pattern matching on FPGAs," in Proc. FPGA'04, Monterey, CA, Feb. 2004, pp. 223-232.

[4] R. Tessier and W. Burleson, "Reconfigurable computing for digital signal processing: A survey," J VLSI Sig Proc Syst, vol. 28, pp. 7-27, May-Jun 2001.

[5] Y. H. Cho and W. H. Mangione-Smith, "Deep network packet filter design for reconfigurable devices," $A C M T$ Embed Comput S, vol. 7, pp. 21:1-21:26, 2008.

[6] J. Villasenor, et al., "Configurable computing solutions for automatic target recognition," in Proc. FCCM'96, Napa Valley, CA, Apr. 1996, pp. 70-79. 
[7] I. Kuon and J. Rose, "Measuring the gap between FPGAs and ASICs," IEEE T Comput Aid D, vol. 26, pp. 203-215, Feb 2007.

[8] I. Sourdis and D. Pnevmatikatos, "Pre-decoded CAMs for efficient and high-speed NIDS pattern matching," in Proc. FCCM'04, Napa, CA, Apr. 2004, pp. 258-267.

[9] K. Pagiamtzis and A. Sheikholeslami, "Contentaddressable memory (CAM) circuits and architectures: A tutorial and survey," IEEE J Solid-St Circ, vol. 41, pp. 712727, Mar 2006.

[10] International Technology Roadmap for Semiconductors, 2007 Edition, Available online at http://www.itrs.net.

[11] W. $\mathrm{Xu}$, et al., "Design of spin-torque transfer magnetoresistive RAM and CAM/TCAM with high sensing and search speed," IEEE T VLSI Syst, vol. 18, pp. 66-74, Jan 2010.

[12] T. Hanyu, et al., "Non-volatile one-transistor-cell multiplevalued CAM with a digit-parallel-access scheme and its applications," Comput Electr Eng, vol. 23, pp. 407-414, Nov 1997.

[13] K. Eshraghian, et al., "Memristor MOS content addressable memory (MCAM): Hybrid Architecture for Future High Performance Search Engines " IEEE T Nanotechnogy, in print, 2011.

[14] S. Matsunaga, et al., "Standby-power-free compact ternary content-addressable memory cell chip using magnetic tunnel junction devices," Appl Phys Express, vol. 2, pp. 023004 , Feb 2009.

[15] K. K. Likharev and D. B. Strukov, "CMOL: Devices, circuits, and architectures," Lecture Notes in Physics, vol. 680, pp. 447-477, 2006.

[16] D. B. Strukov and R. S. Williams, "Four-dimensional address topology for circuits with stacked multilayer crossbar arrays," P Natl Acad Sci USA, vol. 106, pp. 20155-20158, Dec 12009.

[17] D. B. Strukov and K. K. Likharev, "CMOL FPGA: A reconfigurable architecture for hybrid digital circuits with two-terminal nanodevices," Nanotechnology, vol. 16, pp. 888-900, Jun 2005.

[18] D. B. Strukov and K. K. Likharev, "A reconfigurable architecture for hybrid CMOS/nanodevice circuits," in Proc. FPGA'06, Monterey, CA, Feb. 2006, pp. 131-140.

[19] D. Strukov and A. Mishchenko, "Monolithically stackable hybrid FPGA," in Proc. DATE'10, Dresden, Germany, Mar. 2010, pp. 661-666.

[20] K. K. Likharev, "Hybrid CMOS/Nanoelectronic Circuits: Opportunities and Challenges," J Nanoelectron Optoe, vol. 3, pp. 203-230, Dec 2008.

[21] R. Waser, et al., "Redox-based resistive switching memories - nanoionic mechanisms, prospects, and challenges," Adv Mater, vol. 21, pp. 2632-2663, Jul 13 2009.

[22] Y. C. Pershin and M. D. Ventra, "Memory effects in complex materials and nanoscale systems," Advances in Physics, vol. 60, pp. 145-227, 2011.

[23] J. Borghetti, et al., "Electrical transport and thermometry of electroformed titanium dioxide memristive switches," $J$ Appl Phys, vol. 106, art. 124504 , Dec 152009.

[24] J. J. Yang, et al., "Memristive switching mechanism for metal/oxide/metal nanodevices," Nat Nanotechnol, vol. 3, pp. 429-433, Jul 2008.

[25] R. Meyer, et al., "Oxide dual-layer memory element for scalable non-volatile cross-point memory technology," in Proc. Nonvolatile Memory Technology Symposium, Monterey, CA, Nov. 2008, pp. 54-58.

[26] I. G. Baek, et al., "Multi-layer cross-point binary oxide resistve memory (OxRRAM) for post-NAND storage application," in Proc. Electron Device Meeting, Washington, DC, Dec. 2005, pp. 750-753.

[27] L. Tan and T. Sherwood, "Architectures for bit-split string scanning in intrusion detection," IEEE Micro, vol. 26, pp. 110-117, Jan-Feb 2006.

[28] Q. F. Xia, et al., "Memristor-CMOS Hybrid Integrated Circuits for Reconfigurable Logic," Nano Lett, vol. 9, pp. 3640-3645, Oct 2009. 